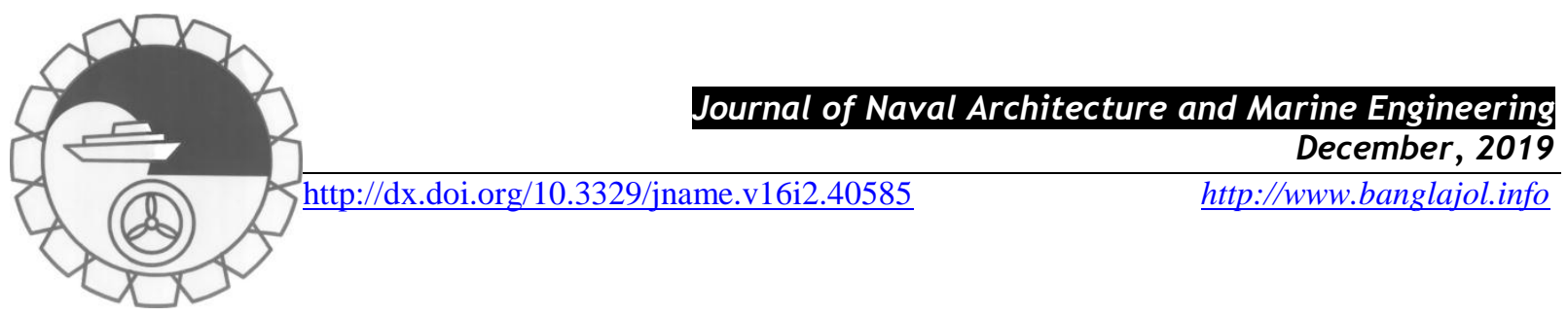

\title{
MIXED CONVECTIVE HYBRID NANOFLUID FLOW IN LID-DRIVEN UNDULATED CAVITY: EFFECT OF MHD AND JOULE HEATING
}

\author{
Ishrat Zahan ${ }^{1}$, R. Nasrin ${ }^{2} *$ and M. A. Alim ${ }^{3}$
}

Department of Mathematics, Bangladesh University of Engineering \& Technology, Dhaka, Bangladesh

${ }^{1}$ Email: ishratzahan@math.buet.ac.bd, $\underline{2 *}$ rehena@math.buet.ac.bd, ${ }^{3}$ maalim@ math.buet.ac.bd

\begin{abstract}
A numerical analysis has been conducted to show the effects of magnetohydrodynamic $(M H D)$ and Joule heating on heat transfer phenomenon in a lid driven triangular cavity. The heat transfer fluid (HTF) has been considered as water based hybrid nanofluid composed of equal quantities of $\mathrm{Cu}$ and $\mathrm{TiO}_{2}$ nanoparticles. The bottom wall of the cavity is undulated in sinusoidal pattern and cooled isothermally. The left vertical wall of the cavity is heated while the inclined side is insulated. The two dimensional governing partial differential equations of heat transfer and fluid flow with appropriate boundary conditions have been solved by using Galerkin's finite element method built in COMSOL Multyphysics. The effects of Hartmann number, Joule heating, number of undulation and Richardson number on the flow structure and heat transfer characteristics have been studied in details. The values of Prandt number and solid volume fraction of hybrid nanoparticles have been considered as fixed. Also, the code validation has been shown. The numerical results have been presented in terms of streamlines, isotherms and average Nusselt number of the hybrid nanofluid for different values of governing parameters. The comparison of heat transfer rate by using hybrid nanofluid, $\mathrm{Cu}$-water nanofluid, $\mathrm{TiO}_{2}$-water nanofluid and clear water has been also shown. Increasing wave number from 0 to 3 enhances the heat transfer rate by $16.89 \%$. The enhanced rate of mean Nusselt number for hybrid nanofluid is found as $4.11 \%$ compared to base fluid.
\end{abstract}

Keywords: Hybrid nanofluid, mixed convection, MHD, Joule heating, undulated cavity, finite element method, heat transfer.

\section{NOMENCLATURE}

$C_{p} \quad$ Specific heat at constant pressure $\left(\mathrm{Jkg}^{-1} \mathrm{~K}^{-1}\right)$

$g \quad$ Gravitational force $\left(\mathrm{ms}^{-2}\right)$

$J \quad$ Joule heating

$k \quad$ Thermal conductivity $\left(\mathrm{Wm}^{-1} \mathrm{~K}^{-1}\right)$

$\mathrm{Ha}$ Hartmann number

$\mathrm{Nu} \quad$ Nusselt number

$p \quad$ Pressure $\left(\mathrm{kgms}^{-2}\right)$

$\mathrm{Pr} \quad$ Prandtl number

$\mathrm{Ri} \quad$ Richardson number

$T \quad$ Dimensional temperature (K)

$u, v \quad$ Dimensional $x$ and $y$ components of velocity $\left(\mathrm{ms}^{-1}\right)$

$U, V \quad$ Dimensionless velocities

$X, Y \quad$ Dimensionless coordinates

$x, y \quad$ Cartesian coordinates (m)

$\begin{array}{ll}\text { Greek Symbols } \\ \beta & \begin{array}{l}\text { Coefficient of thermal expansion } \\ (1 / \mathrm{K})\end{array} \\ \rho & \text { Density }\left(\mathrm{kgm}^{-3}\right) \\ \mu & \text { Dynamic viscosity }\left(\mathrm{Nsm}^{-2}\right) \\ \phi & \text { Weight fraction }(\%) \\ \theta & \text { Dimensionless temperature } \\ \lambda & \text { Number of undulation } \\ \text { Subscripts } \\ f & \text { Fluid } \\ h n f & \text { Hybrid nanofluid } \\ h & \text { Hot } \\ c & \text { Cold } \\ 1 & \text { Copper nanoparticle } \\ 2 & \text { Titanium oxide nanoparticle }\end{array}$

\section{Introduction}

Taking the application of magneto hydrodynamic (MHD) a wide range of applied science, as an example in the field of agriculture, water-magnetic application in static magnetic field influence on growth of plant that plants cells affected by magnetic field can response in unpredictable way according to many factors including species, 1813-8535 (Print), 2070-8998 (Online) @ 2019 ANAME Publication. All rights reserved. Received on: March, 2019 
intensity of magnetic field and exposure period. Application of a magnetic field has been found to be effective in controlling the melt convection during crystal growth from melts under terrestrial conditions and has now been widely used in metal and semiconductor industries. MHD mixed convective flow through a diverging channel, over an inclined plate were studied by Alam et al. (2016) and Ali et al. (2016).

Joule heating is a process in which electrical energy is converted to heat. Common applications of electric heating include space heating, cooking, water heating and industrial processes. Studies of Muhammad et al. (2018), Hossain and Gorla (2013), Nasrin and Alim (2009) were associated with a mixed convection flow of an electrically conducting fluid in the presence of an external magnetic field have received considerable interest due to the enormous applications in various branches of industry, science and technology such as fire engineering, combustion modeling, geophysics, the cooling of nuclear reactors, operation of MHD generators, plasma studies, etc.

Researchers Nasrin (2011), Gangawane and Manikandan (2017) were interested in heat transfer by mixed convection inside lid driven cavities of different shapes as it has huge applications in environment and industry.

Al-Amiri (2007), Agrouaz et al. (2015) described that flow and heat transfer from irregular surfaces are often encountered in many engineering applications to enhance heat transfer such as micro-electronic devices, flatplate solar collectors and flat-plate condensers in refrigerators, flows in the earth's crust, underground cable systems, electric machinery, cooling system of micro-electronic devices, etc. In addition, roughened surfaces could be used in the cooling of electrical and nuclear components where the wall heat flux is known. Surfaces are intentionally roughened sometimes to enhance heat transfer.

One of the proficient passive approaches is using nanofluid in heat transport improvement for enhancing the efficiency of thermal systems like; heat exchangers, thermal storage, solar collectors, photovoltaic/thermal system, biomedical devices, nuclear reactors, engine/vehicle, cooling of electronic components, transformers etc. Several studies have been reported on an MHD convective lid-driven flow of considering different nanofluid under different physical situations. Record of such investigations can be found in the investigations of Jafari et al. (2012), Arani et al. (2012), Zahan and Alim (2018), Zahan et al. (2018) and Ali et al. (2017a-b, 2018).

"Hybrid" nanofluid can be obtained by suspending more than one type of nanoparticles in base fluid. Very recently, hybrid nanofluid, is a gradually mounting study field parallelly to the incessant developments of common nanofluids. Compromised properties between the advantages and disadvantages of the properties of individual nanoparticles are a declared task of hybridization. Moreover, nanoparticles suppliers exhibit noticeable differences in prices of different nanoparticles types. For example, the price of copper nanoparticles is about 10 times greater than that of alumina nanoparticles. Indeed, the "hybrid" nanoparticles should be limited to those prepared as a single composite substance in a base fluid for which their synthetization requires extra attention [Han et al. (2007) Nasrin and Alim (2013), Jan et al. (2007), Chamkha et al. (2017)].

One can use the "hybrid" nanofluid issue to those prepared by mixing unlike nanoparticles with base fluid. The experiments of Ho et al. (2010) conducted an experiment about the mixture of particles of micro-encapsulated phase-change material and alumina nanoparticles in base fluid water. The authors found good agreement between the experimental data of the density and mass fraction and those predicted from the mixture theory. Botha et al. (2011) performed an experiment of hybrid nanofluid based on silver-silica-oil. The authors found more deviated value of the thermal conductivity with the Maxwell (1873) relation at higher solid volume fractions.

In order to remain side by side developments in the extent applications and increasingly developing studies about nanofluids, review papers have been conducted. Hybrid nanoparticles mixture procedure has been reviewed comprehensively by Sarkar et al. (2015), Babu et al. (2017), Kumar and Arasu (2017) and Sidik et al. (2016). This review showed the arithmetical models of hybrid nanofluids properties. The authors Zahan et al. (2019), Bumataria et al. (2019), Ben-Cheikh et al. (2013) and Ismael et al. (2014) performed current research aspects in mono as well as hybrid nanofluids based on different physical models at different conditions to show heat transfer mechanism.

Above literature shows that many research have been done on these keywords like mixed convection, lid-driven sinusoidal cavity, hybrid nanofluid, MHD and Joule heating. But the research in combination of all these important factors has not been conducted yet. Mixed convection in a lid-driven triangular sinusoidal enclosure filled with such hybrid nanofluid in presence of MHD and Joule heating depending on the existing mathematical 
formulae needs additional attention to disclose its performance in this significant field. Specific applications of the present numerical research are micro-electronic devices, flat-plate solar collectors, flat-plate condensers in refrigerators, flows in the earth's crust, underground cable systems, electric machinery and cooling system of micro-electronic devices etc. The objective of this research paper is to show the combined effects of MHD and Joule heating on hybrid nanofluid mixed convective flow inside lid-driven sinusoidal triangular enclosure.

\section{Problem Formulation}

The schematic diagram of the studied configuration has been depicted in Figure 1. It consist of a two dimensional vertical lid-driven triangular enclosure of side length $H$. The left surface has been assigned to temperature $T_{h}$ while the sinusoidal wavy bottom surface of the enclosure has been cooled at a constant temperature $T_{c}$. Under all circumstances $T_{h}>T_{c}$ condition has been maintained. The inclined surface of the triangular cavity has been thermally insulated. Furthermore, the left wall has been assumed to slide in its own plane at a constant speed $v_{0}$. A uniform external magnetic field has been applied in the horizontal direction normal to the vertical wall. The heat transfer medium has been taken as electrically conducting water based hybrid nanofluid consisting equal solid volume fraction of $\mathrm{TiO}_{2}$ and $\mathrm{Cu}$ nanoparticles. It has been also assumed that both the fluid and nanoparticles are in thermal equilibrium and there is no slip between them. The hybrid nanofluid used in the analysis has been considered as laminar and incompressible. The gravitational acceleration acts to the vertical downward surface. Thermo-physical properties of base fluid and different nanoparticles have been shown in Table 1 and taken from Rashad et al. (2018) and Nasrin et al. (2013), assumed constant except for the density variation, which is maintained on Boussinesq approximation.

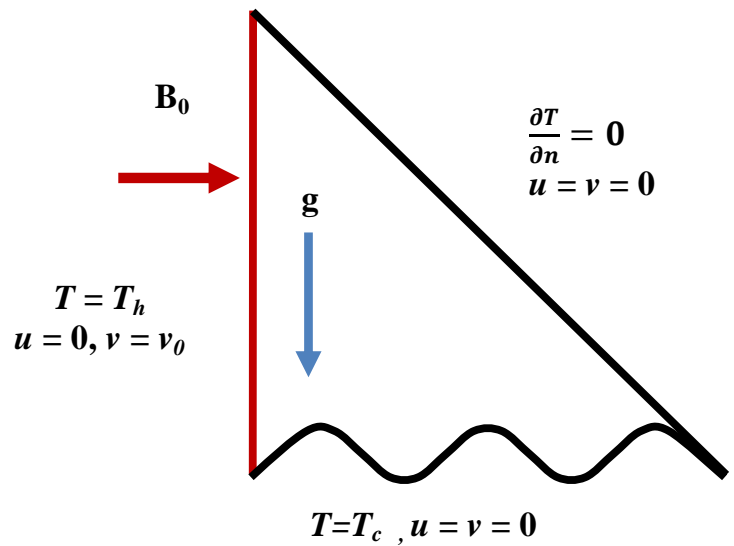

Figure 1: Physical model

Table 1: Thermo-physical properties [Rashad et al. (2018) and Nasrin et al. (2013)]

\begin{tabular}{cccc}
\hline Physical Properties & Fluid Phase (Water) & Cu & TiO $_{2}$ \\
\hline$C_{p}(\mathrm{~J} / \mathrm{kgK})$ & 4179 & 385 & 686.2 \\
$\rho\left(\mathrm{kg} / \mathrm{m}^{3}\right)$ & 997.1 & 8933 & 4250 \\
$k(\mathrm{~W} / \mathrm{mK})$ & 0.6 & 401 & 8.9538 \\
$\beta \times 10^{-5}(1 / \mathrm{K})$ & 21 & $1.67 \times 10^{-5}$ & $0.9 \times 10^{-5}$ \\
$\sigma(\mu \mathrm{S} / \mathrm{cm})$ & 0.05 & $5.96 \times 10^{7}$ & $6.28 \times 10^{-5}$ \\
\hline
\end{tabular}

\section{Mathematical Modeling}

The 2D numerical simulation has been performed in steady state conditions. The governing partial differential equations (dimensional form) in terms of laminar flow and thermal energy equations using hybrid nanofluid have been given below: 


$$
\begin{aligned}
& \frac{\partial u}{\partial x}+\frac{\partial v}{\partial y}=0 \\
& \left(u \frac{\partial u}{\partial x}+v \frac{\partial u}{\partial y}\right)=-\frac{1}{\rho_{h n f}} \frac{\partial p}{\partial x}+\frac{\mu_{h n f}}{\rho_{h n f}}\left(\frac{\partial^{2} u}{\partial x^{2}}+\frac{\partial^{2} u}{\partial y^{2}}\right) \\
& \left(u \frac{\partial v}{\partial x}+v \frac{\partial v}{\partial y}\right)=-\frac{1}{\rho_{h n f}} \frac{\partial p}{\partial y}+\frac{\mu_{h n f}}{\rho_{h n f}}\left(\frac{\partial^{2} v}{\partial x^{2}}+\frac{\partial^{2} v}{\partial y^{2}}\right)-\frac{\sigma_{h n f}}{\rho_{h n f}} B_{0}^{2} v+\frac{(\rho \beta)_{h n f}}{\rho_{h n f}} \frac{g H}{v_{0}}\left(T-T_{c}\right) \\
& \left(u \frac{\partial T}{\partial x}+v \frac{\partial T}{\partial y}\right)=\alpha_{h n f}\left(\frac{\partial^{2} T}{\partial x^{2}}+\frac{\partial^{2} T}{\partial y^{2}}\right)+\frac{\alpha_{h n f}}{\left(\rho C_{p}\right)_{h n f}} B_{0}^{2} u^{2}
\end{aligned}
$$

The above equations have been non-dimensionalized using the following dimensionless quantities:

$$
X=\frac{x}{H}, \quad Y=\frac{y}{H}, U=\frac{v_{0} H}{\alpha_{f}}, \quad V=\frac{v_{0} H}{\alpha_{f}}, \quad \theta=\frac{T-T_{c}}{T_{h}-T_{c}}, P=\frac{p H^{2}}{\rho_{f} \alpha_{f}^{2}}
$$

The shape of the bottom wavy surface profile has been assumed to mimic the following pattern $Y=A[1-\cos (2 \lambda \pi X)]$

where $A$ is the dimensionless amplitude of the wavy surface and $\lambda$ is the number of undulation.

Using equation (5) the governing partial differential equations (1-4) have been transformed into nondimensional form according to Rashad et al. (2018) and Nasrin et al. (2013):

$$
\begin{aligned}
& \frac{\partial U}{\partial X}+\frac{\partial V}{\partial Y}=0 \\
& \left(U \frac{\partial U}{\partial X}+V \frac{\partial U}{\partial Y}\right)=-\frac{\partial P}{\partial X}+\frac{\rho_{f}}{\rho_{h n f}} \frac{\mu_{h n f}}{\mu_{f}} \operatorname{Pr}\left(\frac{\partial^{2} U}{\partial X^{2}}+\frac{\partial^{2} U}{\partial Y^{2}}\right) \\
& \left(U \frac{\partial V}{\partial X}+V \frac{\partial V}{\partial Y}\right)=-\frac{\partial P}{\partial Y}+\frac{\rho_{f}}{\rho_{h n f}} \frac{\mu_{h n f}}{\mu_{f}} \operatorname{Pr}\left(\frac{\partial^{2} V}{\partial X^{2}}+\frac{\partial^{2} V}{\partial Y^{2}}\right)-\frac{\rho_{f}}{\rho_{h n f}} \frac{\sigma_{h n f}}{\sigma_{f}} H a^{2} \operatorname{Pr} V+\frac{(\rho \beta)_{h n f}}{(\rho \beta)_{f}} \operatorname{Ri\theta } \\
& \left(U \frac{\partial \theta}{\partial X}+V \frac{\partial \theta}{\partial Y}\right)=\frac{\alpha_{h n f}}{\alpha_{f}}\left(\frac{\partial^{2} \theta}{\partial X^{2}}+\frac{\partial^{2} \theta}{\partial Y^{2}}\right)+\frac{\alpha_{h n f}}{\alpha_{f}} \frac{\left(\rho C_{p}\right)_{f}}{\left(\rho C_{p}\right)_{n f}} J U^{2}
\end{aligned}
$$

where, $\operatorname{Pr}=\frac{\mu_{f} C_{p f}}{k_{f}}, \quad R i=\frac{g \beta_{f} \Delta T H}{v_{0}}, \quad H a=B_{0} H \sqrt{\sigma_{f} / \mu_{f}}$ and $J=\frac{\sigma_{f} H^{2} v_{f} \sqrt{G r}}{\left(\rho C_{p}\right)_{f} \Delta T}$ be the Prandtl number,

Richardson number, Hartmann number and Joule heating, respectively.

The boundary conditions imposed on the flow have been listed as:

On the left wall: $U=0, V=1, \theta=1$

On the inclined wall: $U=V=0, \frac{\partial \theta}{\partial n}=0$

On the wavy bottom wall: $U=V=0, \theta=0$

where $n$ be the distances either along $x$ or $y$ directions acting normal to the inclined surface respectively. The following models of effective properties of hybrid nanofluids have been chosen as:

thermal diffusivity $\alpha_{h n f}=k_{\text {hnf }} /\left(\rho C_{p}\right)_{h n f}$

density, $\rho_{\text {hnf }}=(1-\phi) \rho_{f}+\phi_{1} \rho_{1}+\phi_{2} \rho_{2}$

heat capacitance, $\left(\rho C_{p}\right)_{h n f}=(1-\phi)\left(\rho C_{p}\right)_{f}+\phi_{1}\left(\rho C_{p}\right)_{1}+\phi_{2}\left(\rho C_{p}\right)_{2}$

thermal expansion coefficient, $(\rho \beta)_{h n f}=(1-\phi)(\rho \beta)_{f}+\phi_{1}(\rho \beta)_{1}+\phi_{2}(\rho \beta)_{2}$

specific heat at constant pressure, $C_{p h n f}=\frac{(1-\phi)\left(\rho C_{p}\right)_{f}+\phi_{1}\left(\rho C_{p}\right)_{1}+\phi_{2}\left(\rho C_{p}\right)_{2}}{(1-\phi) \rho_{f}+\phi_{1} \rho_{1}+\phi_{2} \rho_{2}}$ 
viscosity of Brinkman model (1952) $\mu_{h n f}=\frac{\mu_{f}}{\left(1-\phi_{1}-\phi_{2}\right)^{2.5}}$

thermal conductivity of Maxwell-Garnett model (1873)

$$
k_{h n f}=k_{f} \frac{\frac{\left(\phi_{1} k_{1}+\phi_{2} k_{2}\right)}{\phi}+2 k_{f}+2\left(\phi_{1} k_{1}+\phi_{2} k_{2}\right)-2 \phi k_{f}}{\frac{\left(\phi_{1} k_{1}+\phi_{2} k_{2}\right)}{\phi}+2 k_{f}-\left(\phi_{1} k_{1}+\phi_{2} k_{2}\right)+\phi k_{f}}
$$

and electrical conductivity of Maxwell-Garnett model (1873)

$$
\frac{\sigma_{h n f}}{\sigma_{f}}=1+\frac{3\left\{\frac{\left(\phi_{1} \sigma_{1}+\phi_{2} \sigma_{2}\right)}{\sigma_{f}}-\left(\phi_{1}+\phi_{2}\right)\right\}}{\left\{\frac{\left(\phi_{1} \sigma_{1}+\phi_{2} \sigma_{2}\right)}{\phi \sigma_{f}}+2\right\}-\left\{\frac{\left(\phi_{1} \sigma_{1}+\phi_{2} \sigma_{2}\right)}{\sigma_{f}}-\left(\phi_{1}+\phi_{2}\right)\right\}}
$$

where $\phi$ is the overall volume concentration of two different types of nanoparticles dispersed in hybrid nanofluid and is calculated as $\phi=\phi_{1}+\phi_{2}$

The mean Nusselt number at the left heated wall of the cavity is $N u=-\frac{k_{h n f}}{k_{f}} \int_{0}^{H} \frac{\partial \theta}{\partial X} d Y$ where $H$ is the length of the heated surface.

\section{Computational Procedure}

Using the Galerkin weighted residual finite element technique of Dechaumphai (1999), Taylor and Hood (1973) the momentum and energy balance equations have been solved using COMSOL Multyphysics. In this method, the solution domain has been discretized into finite element meshes, which have been composed of non-uniform triangular elements. Then the nonlinear governing partial differential equations have been transferred into a system of integral equations by applying Galerkin weighted residual method. The basic unknowns for the governing partial differential equations (7-10) are the velocity components $U, V$, the temperature $\theta$ and the pressure $P$. The six nodes with triangular element have been used in this numerical research. All six nodes have been associated with velocities as well as temperature while three corner nodes with pressure. The nonlinear algebraic equations so obtained have been modified by imposition of boundary conditions. These modified nonlinear equations have been transferred into linear algebraic equations by using Newton's method. Finally, these linear equations have been solved by using triangular factorization method. The convergence criterion for the solution procedure has been defined as $\left|\psi^{n+1}-\psi^{n}\right| \leq 10^{-6}$, where $n$ is the number of iteration and $\psi$ is a function of $U, V$ and $\theta$.

Rashad et al. (2018)
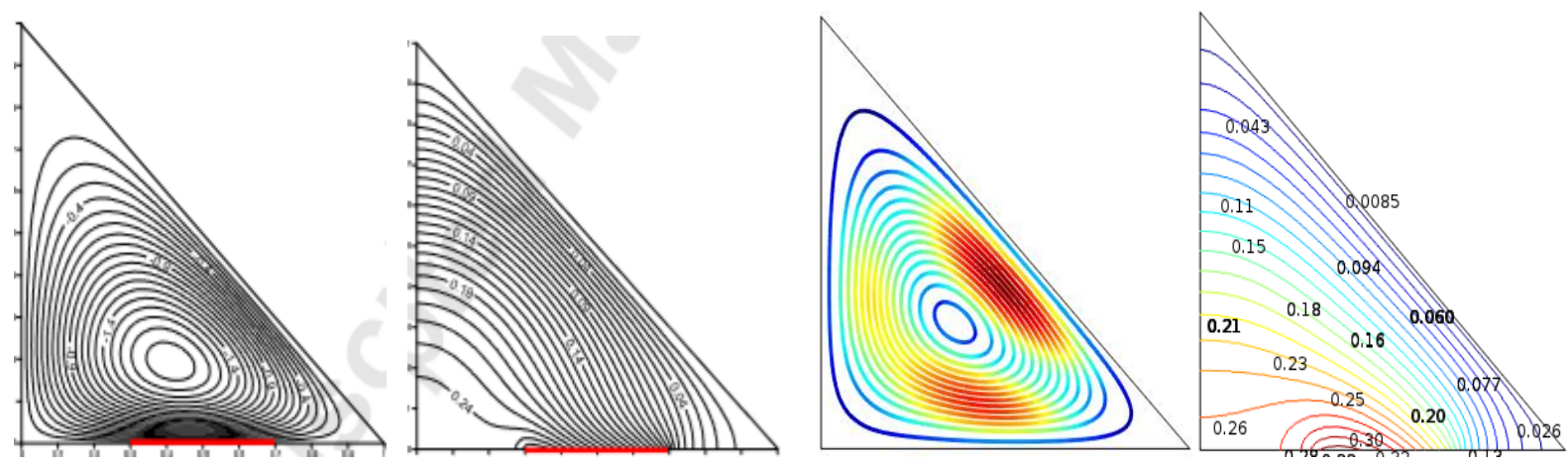

Figure 2: Code validation of the streamlines and isotherms between Rashad et al. (2018) and that of present research at $H a=10, \phi=0.05, D=0.5, R a=10^{4}, Q=1, B=0.4$ 


\subsection{Code validation}

In order to authenticate the exactness of present numerical technique, the obtained results in special cases have been compared with the results obtained by Rashad et al. (2018). These comparisons have been presented obviously in Figure 2 in terms of the streamlines and Isotherms. The code validation has been conducted while employing the dimensionless parameters as $H a=10, \phi=0.05, D=0.5, R a=10^{4}, Q=1, B=0.4$. A very good agreement has been found between the present results and the results of Rashad et al. (2018). These flattering comparisons provide confidence in the numerical results to be reported subsequently.

\subsection{Mesh generation}

Meshing the complex geometry create the finite element method a potential technique to solve boundary value problems occurring in a range of engineering applications. It is basically a discrete representation of the geometric domain on which the problem is to be calculated. Figure 3 shows the mesh configuration of present physical domain with triangular finite elements.

\subsection{Grid sensitivity test}

In order to determine the proper grid size for this study, a grid independence test is conducted with five types of mesh for $\operatorname{Re}=100, \operatorname{Pr}=6.2, \operatorname{Ri}=1.0, \mathrm{Ha}=10, \mathrm{~J}=1$ and $\lambda=3$ which is shown in Figure 4. Corresponding grid densities are 7182 nodes, 592 elements, 29 [s] ; 11062 nodes, 1133elements, 42 [s]; 15074 nodes, 1744 elements, 64 [s]; 18082 nodes, 4484 elements, 105 [s]; and 29726 nodes, 12502 elements, 255 [s]. The extreme value of $\mathrm{Nu}$ is used as the monitoring variable for sensitivity measure of the accuracy of the solution. Taking into account both the precision of numerical values and computational time, the present calculations have been performed with 18082 nodes and 4484 elements grid system. From Figure 4, one can observe that no further improvement in accuracy occur using higher number of elements.

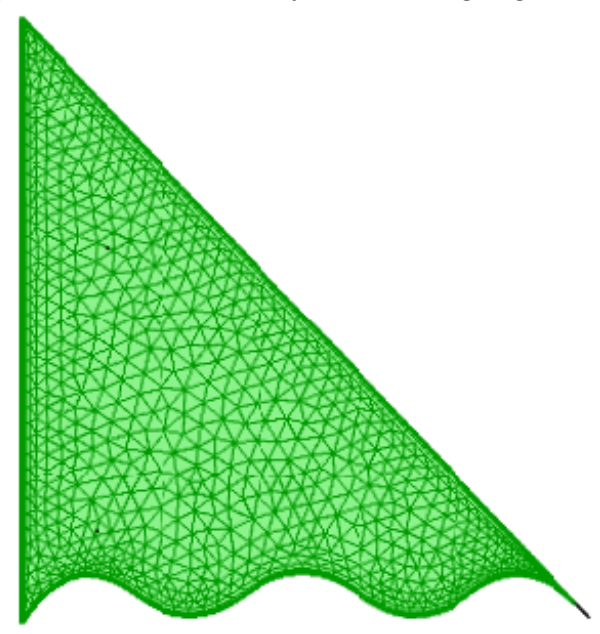

Figure 3: Mesh configuration of elements for the lid

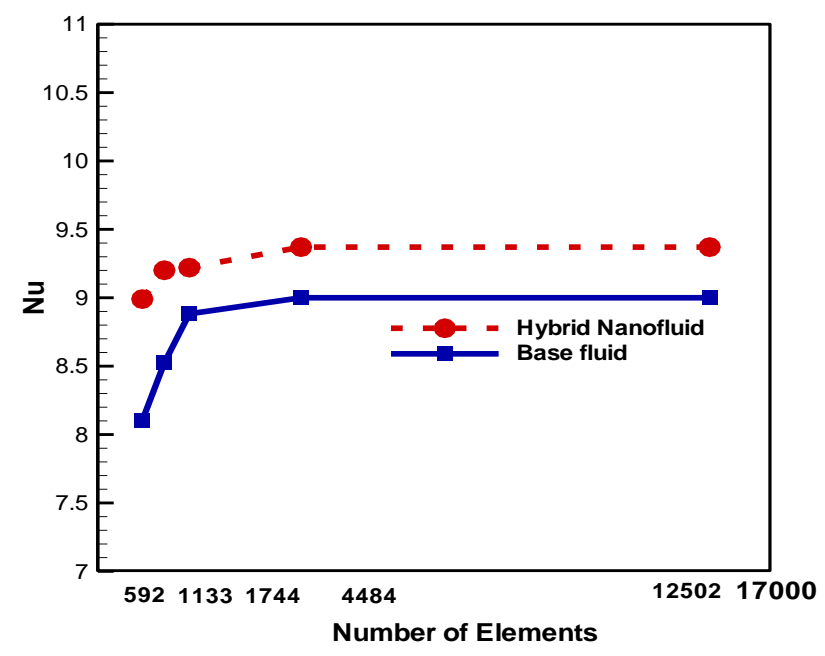

Figure 4: Grid independence check

\section{Results and Discussion}

The characteristics of the flow and temperature fields in the lid-driven cavity have been examined by exploring the effects of the Richardson number $(R i)$, Hartmann number $(H a)$ and Joule heating parameter $(J)$ as a function of number of undulations $(\lambda)$. Such variables have been examined through the steady state version of the streamline and temperature distributions as well as the average Nusselt number $(\mathrm{Nu})$. In the current numerical investigation, the following parametric domains of the dimensionless groups have been considered: $0.5 \leq R i \leq 10,0 \leq H a \leq 50,0 \leq J \leq 2$ and $0 \leq \lambda \leq 3$. The Prandtl number $\operatorname{Pr}=6.2$ has been measured throughout the simulation with $5 \%$ solid volume fraction of the nanoparticles. 


\subsection{Effect of Richardson number}

In heat transfer problems, Richardson number represents the importance of the effect of forced to natural convection phenomena. Due to the influence of buoyancy force if the Richardson number is much less than unity, convection is unimportant in the flow. If it is much greater than unity, buoyancy is dominant the flow behavior. Figure 5(a) provides the information about the sensitivity of the streamlines pattern due to the variation of Richardson number $(R i)$ and number of undulations $(\lambda=0,1,2,3)$ for $\mathrm{Ha}=10, J=1, \operatorname{Pr}=6.2$ with the volume fraction of the suspended naoparticles $\phi=5 \%$. As mentioned before the magnitude of $\phi$ composes of equal quantities of $\mathrm{Cu}$ and $\mathrm{TiO}_{2}$ nanoparticles. From the streamlines contours it is found that for purely forced convection $(R i=0.5)$ there exist a clockwise upward circulating cell which turns to the cold inclined wall and falls down to form a weak primary egg shape eddy near the heated partition created by the movement of the liddriven wall. This is due to the dominance of forced convection in the flow regime. In this case, the streamlines are almost identical for all values of $\lambda$. At combined convection regime $(R i=1.0)$ it can be seen that shape of the vortex spread in size and the corresponding flow domain convert solely to free convection regime. On the other hand increasing $R i$ up to 10 causes the nonofluid to circulate strongly and occupies all most area of the cavity space in wavy form owing to the presence of sinusoidal surface. This is logical because escalating the parameter $R i$ assists buoyancy flow, hence the natural convection mode because buoyancy often plays a significant role in defining the mixed convection flow. But raising the value of $\lambda$ the magnitude of the flow vortex becomes weaken for all value of $R i$. Because on mounting the value of $\lambda$ the fluid moves smoothly up and down, then there produces friction between the wall and the fluid which creates heat and slow down the fluid velocity due to roughness of the bottom surface. In this case, the strength of the natural convection dominates the flow regime. It is also notice that with the increasing values of $\lambda$, cavity core fills with more streamlines and minor vortices form near the left side as the increased number of waves retards the fluid motion in the bottom portion of the cavity.

The influence of $R i$ on temperature field has been plotted in the Figure 5(b). From this figure, it is noticed that for $R i=0.5$, the isothermal line bifurcate with the plume-like behavior. In this situation, it is also found that the isotherms depart from the middle section of the enclosure and try to get crowded on the bottom wavy surface due to the influence of forced convection. Subsequently, the thermal current activity is influenced firmly by the conductivity properties of hybrid nanofluid. There is no major distinction in the isothermal lines for $R i=0.5$ in compare to $R i=1$ except the isothermal contours are clustered near the heated vertical wall of the cavity. For a fixed value of $\lambda$, the thermal field has a little change with the variation in $R i$. Isothermal lines become slightly denser near the bottom corner of the heated surface at higher values of $R i$. Isothermal lines get the wavy pattern due to the waviness of the surface. Escalating the convective parameter $R i=10$ steeper temperature gradient near the heated wall is evident and the isothermal lines get the parabolic shape near the inclined cold wall. In the considered regime, the buoyancy effect balances the flow patterns and a thin boundary layer is developed close to the bottom wavy surface. It is also captured that the mostly horizontal isothermal lines, occupy in the lower section of the cavity. Furthermore, the thermal boundary layer near the underside surface becomes slightly less parallel and concentrates when the number of wave increases.

\subsection{Effect of Hartmann number}

The influence of Hartmann number and wave number on the flow pattern has been depicted in the Figure 6(a) for $\operatorname{Pr}=6.2, J=1, R i=1$ and $\phi=0.05$. In the absence of magnetic field $(H a=0)$, the fluid flow is described by a tiny revolving cell with vortex at the centre generated by the movement of the upper lid. The vortex of the cell becomes weaker with mounting the magnetic field strength from 0 to 50. Magnetic field creates Lorentzs force which opposes the buoyancy forces. Due to this effect fluid motion is slowed down in the bulk of the cavity. For this reason, the vortex related with $H a=0$ circulates generously within most of the cavity, while with the boost in the value of $\mathrm{Ha}$, the magnetic force quantifies and shrinks the vortex of the fluid flow. It means that the magnetic field strongly affects the fluid flow. For $H a=50$ and escalating $\lambda$ from 1 to 3 the size of the petite vortex raises adjacent to the hot wall. With the increasing values of $\lambda$, cavity core fills with more streamlines. However, from the streamline contour it is found that for a particular Hartmann number, deviation in $\lambda$ causes little change in streamlines. The flow outline remains nearly similar except the central domain changes its shape from circular to oval for all higher values of $\mathrm{Ha}$. 

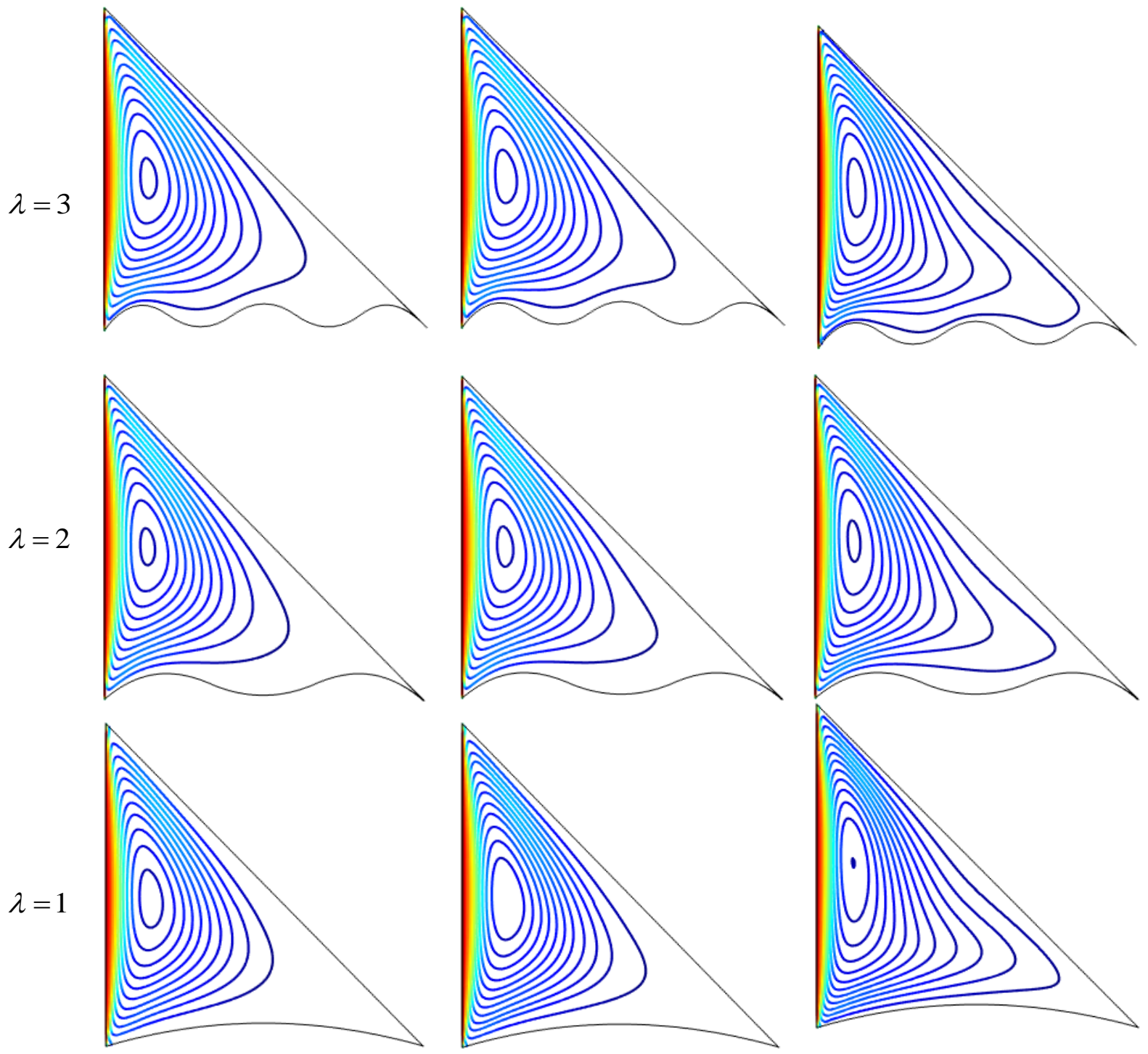

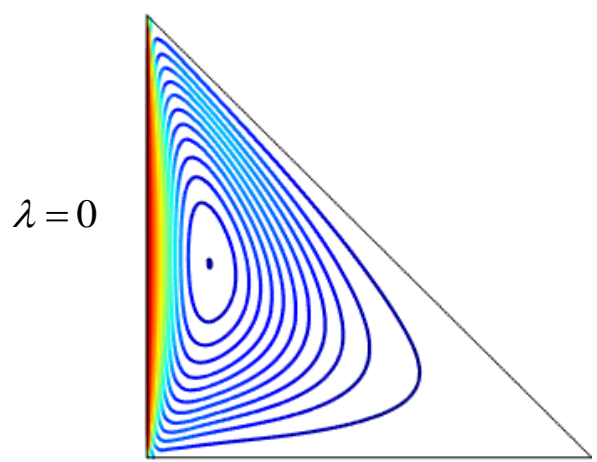

$R i=0.5$

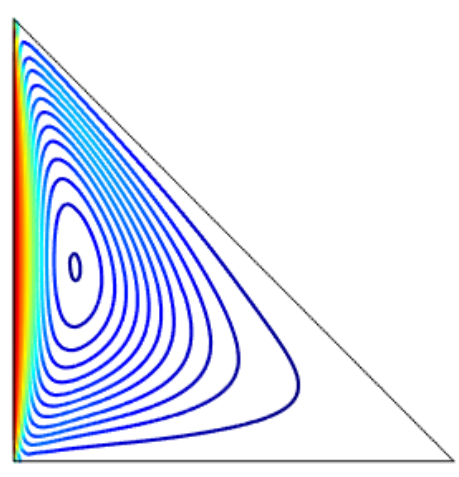

$R i=1$

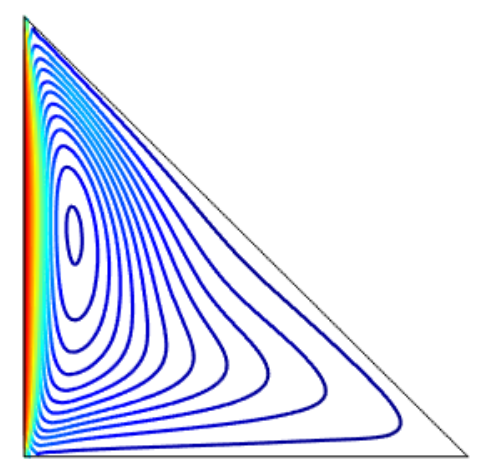

$R i=10$

Figure 5(a): Streamlines for various $R i$ and $\lambda$ (at $H a=10, J=1, \operatorname{Pr}=6.2$ and $\Phi=5 \%$ ) 


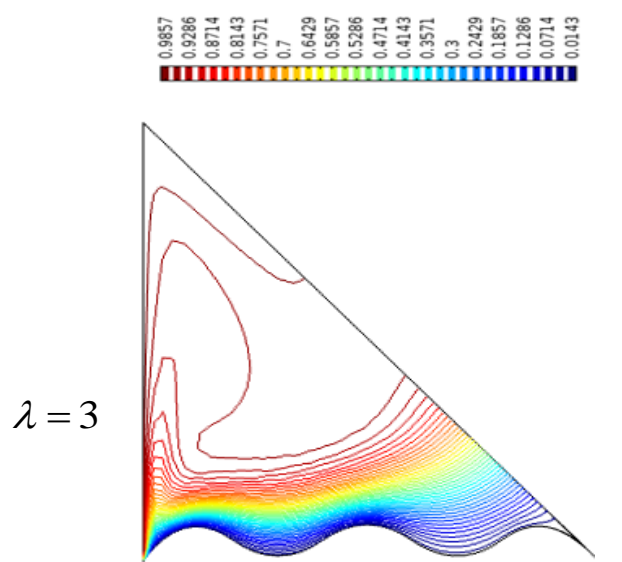

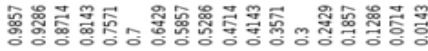

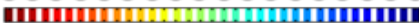
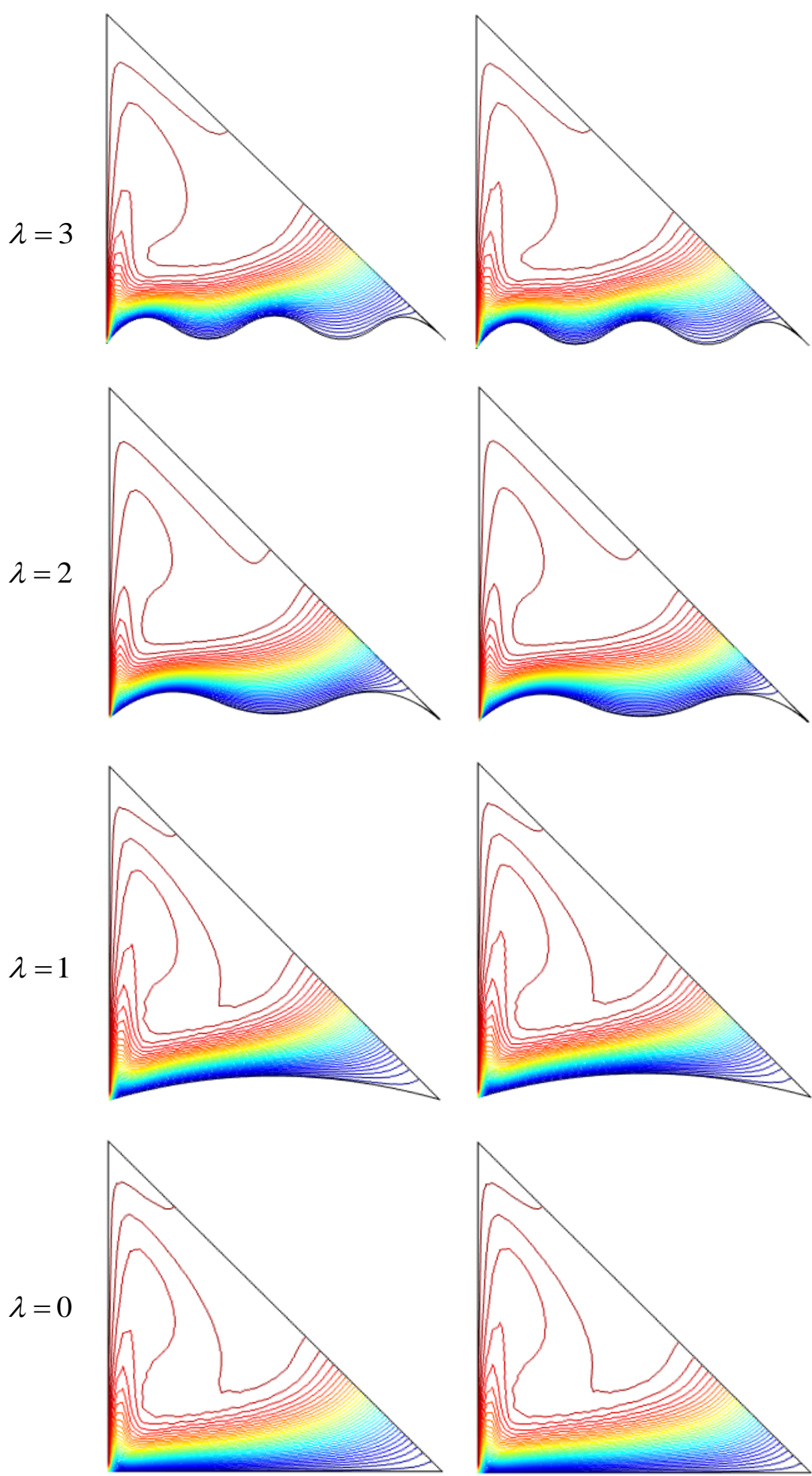

$R i=0.5$
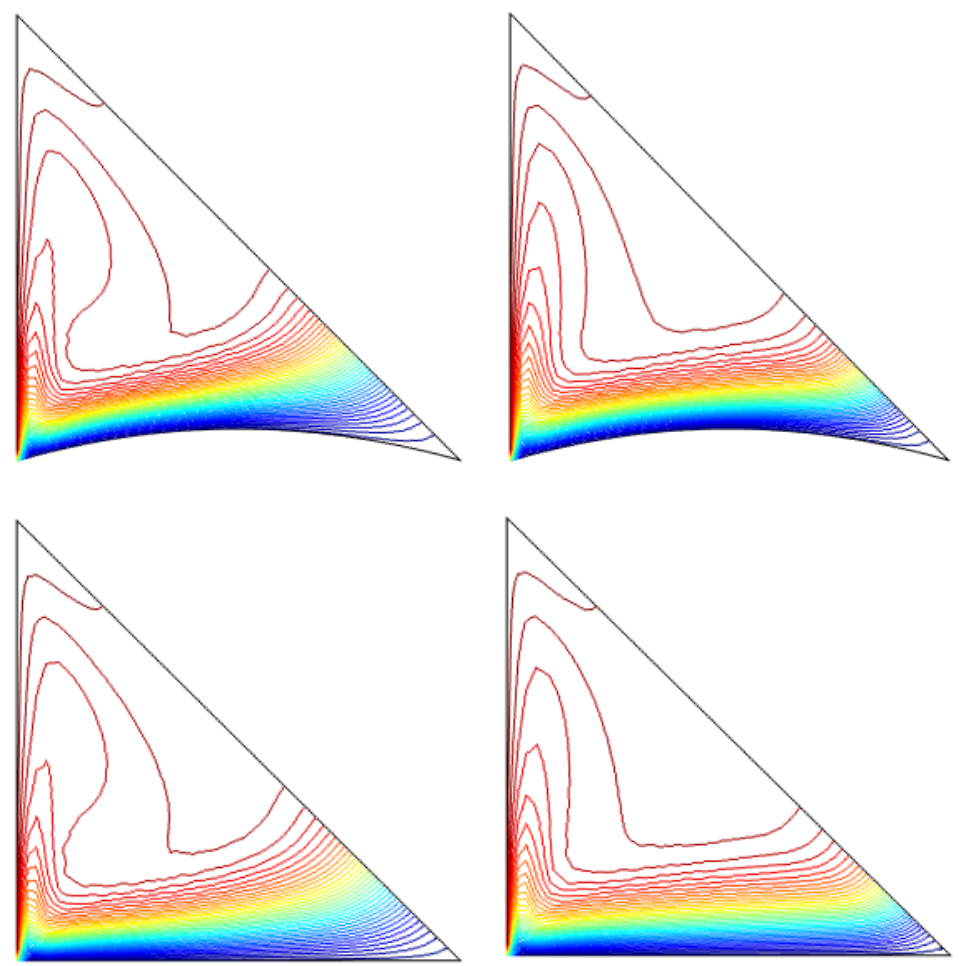

$R i=1$

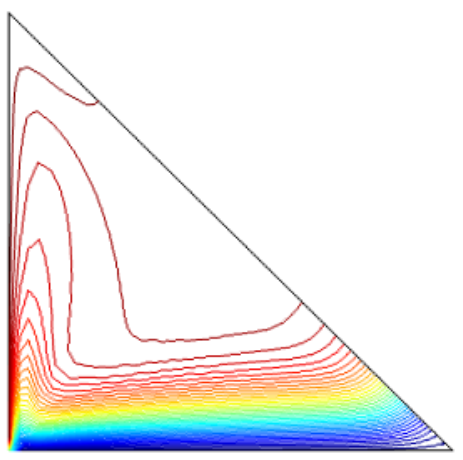

$R i=10$

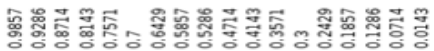

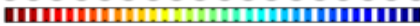
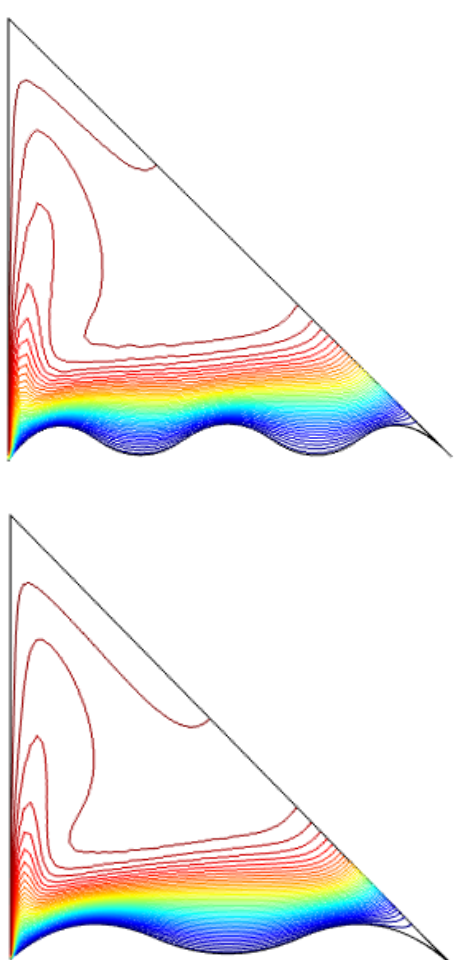

Figure 5(b): Isothermal lines for various $R i$ and $\lambda$ (at $H a=10, \operatorname{Pr}=6.2, J=1$ and $\Phi=5 \%$ ) 
I. Zahan, R. Nasrin and M.A. Alim/ Journal of Naval Architecture and Marine Engineering, 16(2019) 109-126
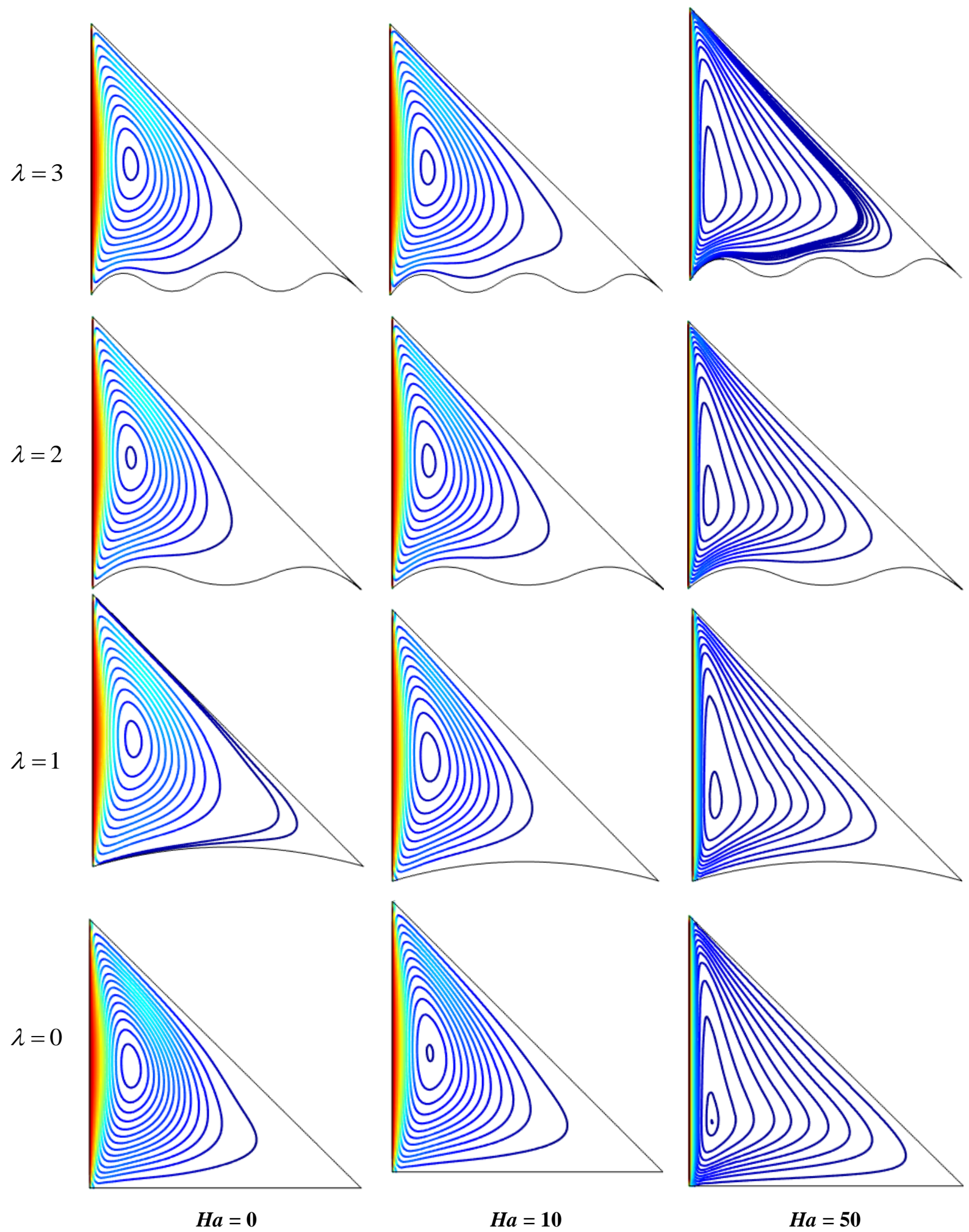

Figure 6(a): Streamlines for various $H a$ and $\lambda$ (at $R i=1, \operatorname{Pr}=6.2, J=1$ and $\Phi=5 \%$ )

Mixed convective hybrid nanofluid flow in lid-driven undulated cavity: effect of MHD and Joule heating

118 


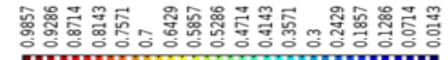

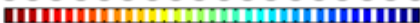
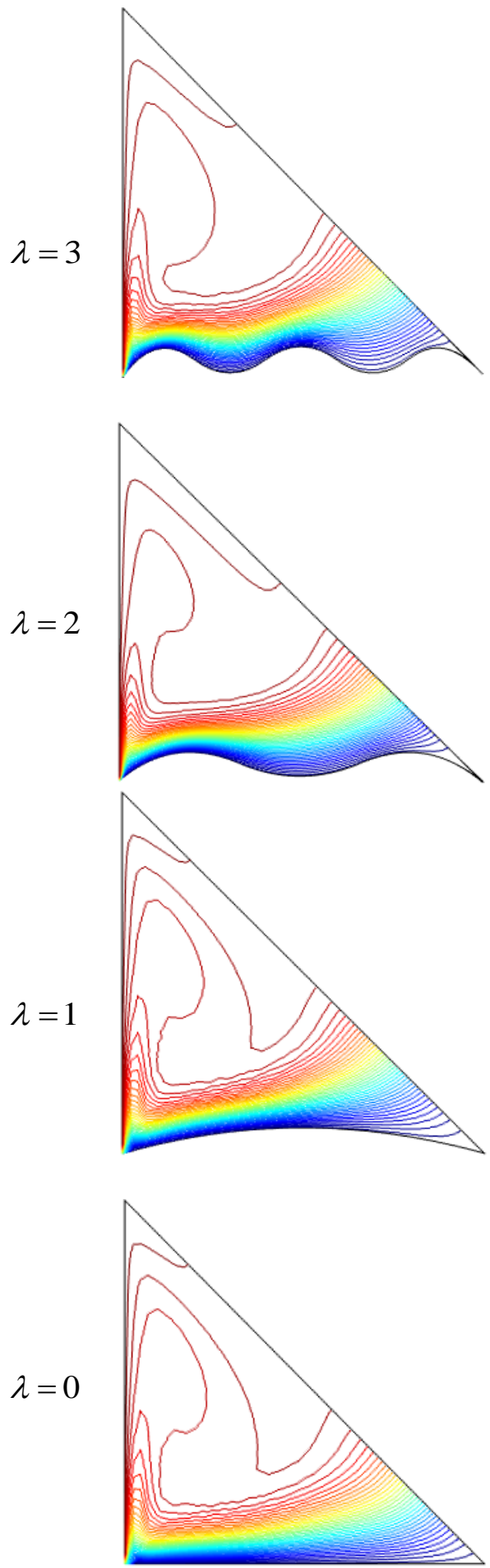

$H a=0$

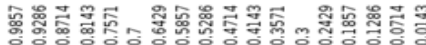

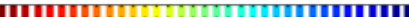
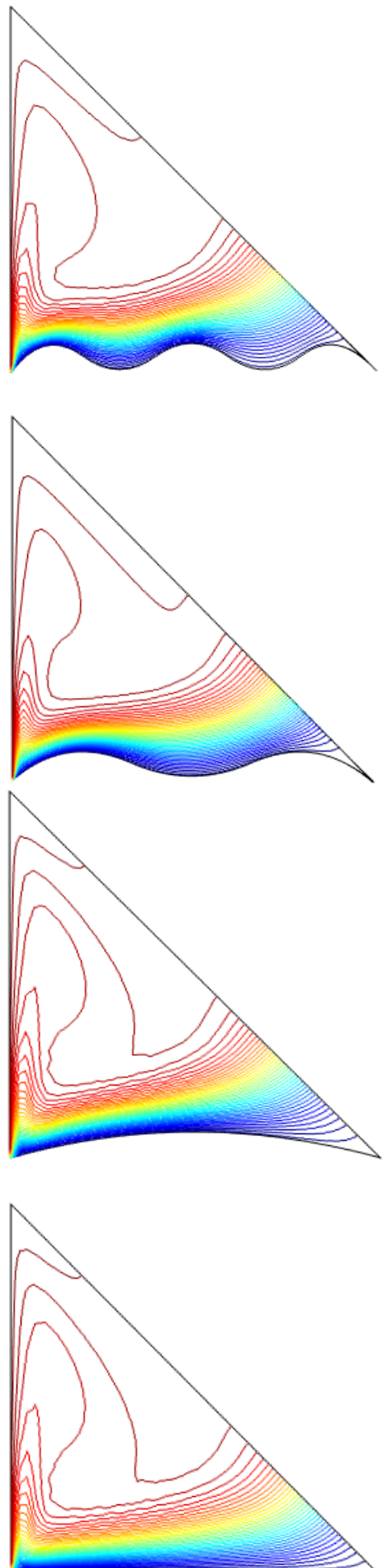

$H a=10$

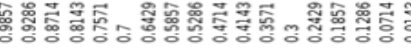

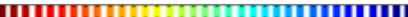
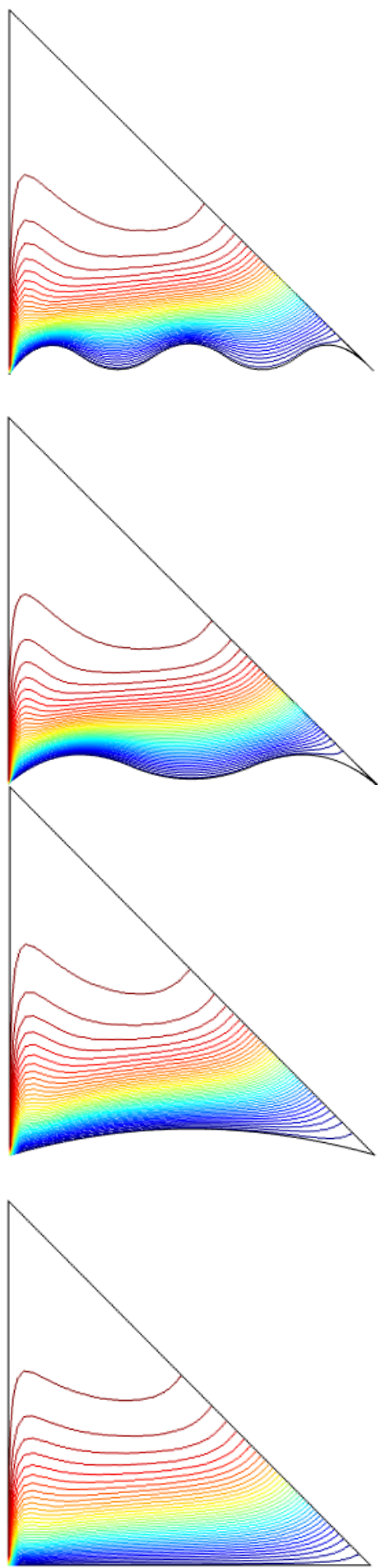

$H a=50$

Figure 6(b): Isothermal lines for various $H a$ and $\lambda$ (at $R i=1.0, J=1, \operatorname{Pr}=6.2$ and $\Phi=5 \%$ ) 

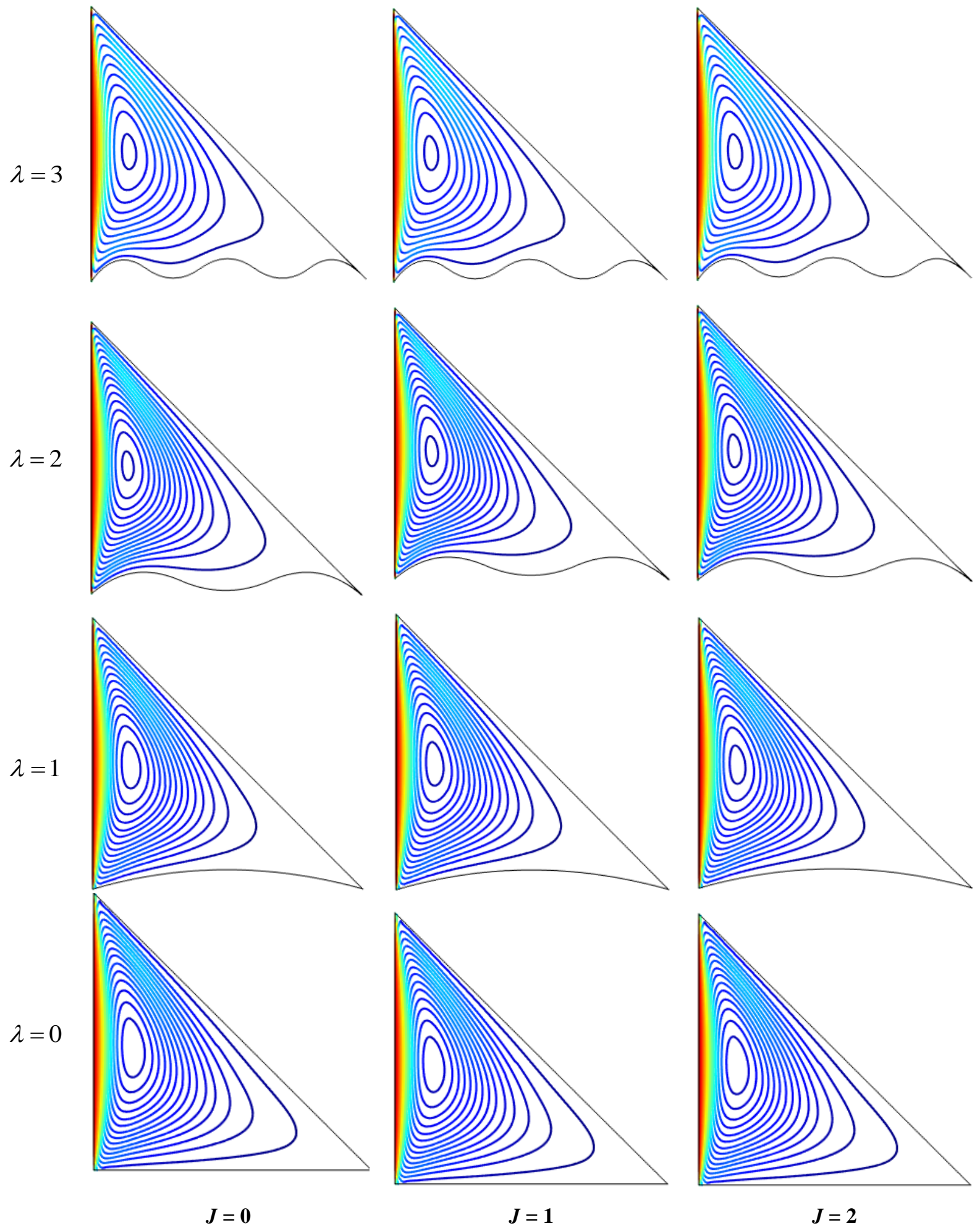

Figure 7(a): Streamlines for various $J$ and $\lambda$ (at $R i=1.0, \operatorname{Pr}=6.2, H a=10$ and $\Phi=5 \%$ ) 


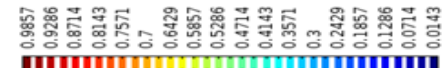
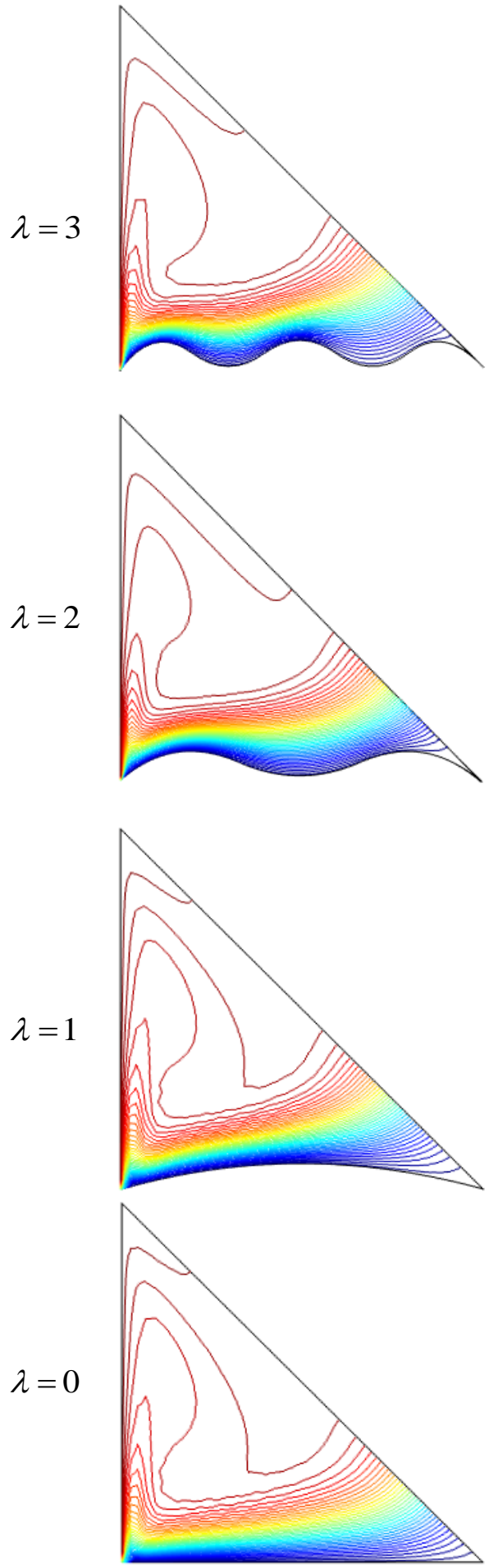

$J=0$

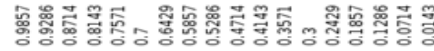

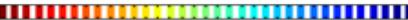
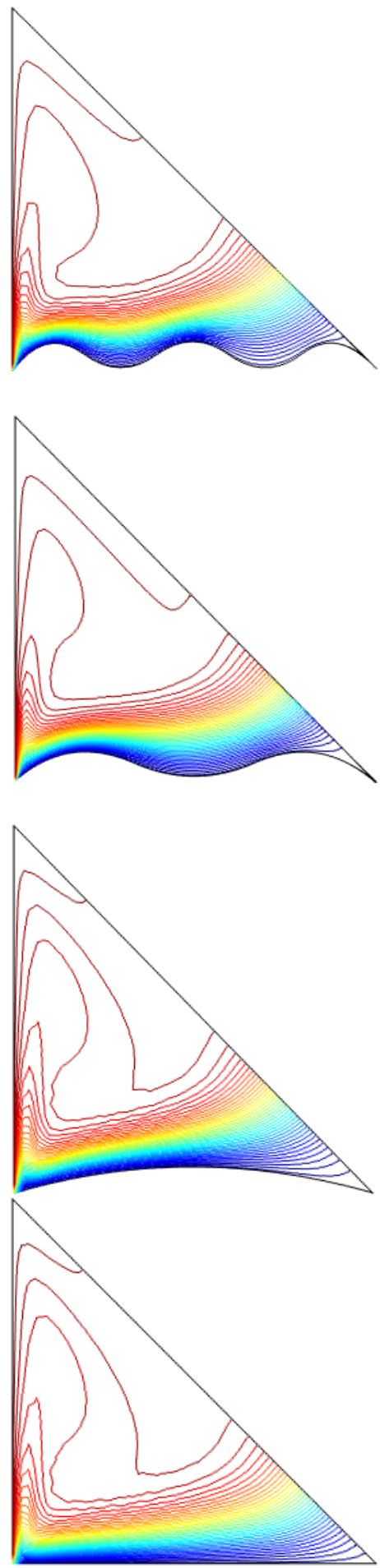

$J=1$

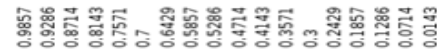

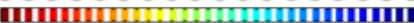
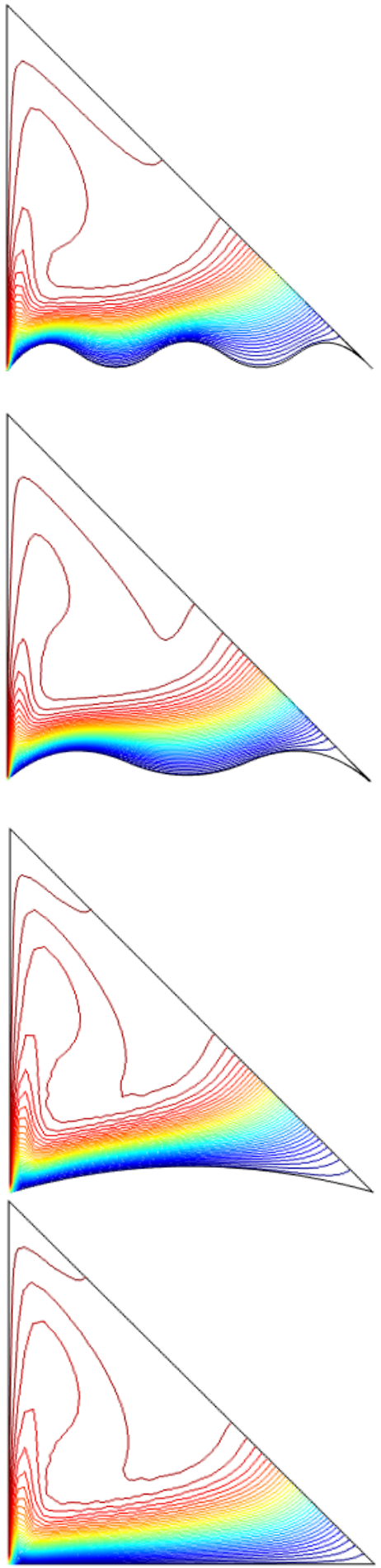

$J=\mathbf{2}$

Figure 7(b): Isothermal lines for various $J$ and $\lambda(R i=1.0, P r=6.2, H a=10$ and $\Phi=5 \%)$ 
Figure 6(b) presents the temperature field for different values of $H a$ and $\lambda$. Higher magnetic field increases internal friction of the fluid which creates some heat in the fluid. It is observed for $\mathrm{Ha}=0$, most of the isothermal lines occupy all the cavity space with an initiation of the plume-like behavior. But at higher Hartmann number up to 50 these lines move from the heated moving wall analogously to the bottom wavy surface towards the middle section of the cavity. These phenomena are seen for all values of wave numbers. On the other hand, for any particular $H a$, high temperature gradient is seen at $\lambda=3$ than other values of $\lambda$. Consequently, the Isothermal lines become slightly denser near the wavy surface at low Hartmann number and higher values of $\lambda$. These lines are less bended for higher magnetic field effect.

\subsection{Effect of Joule heating}

The flow field in terms of streamlines for three representative values of the Joule heating parameter $(0,1$ and 2$)$ and wave number $(0,1,2$ and 3 ) has been displayed in the Figure 7(a) for $\operatorname{Pr}=6.2, H a=10, R i=1$ and $\phi=$ 0.05 . Electrically conducting fluid releases some heat known as Joule heating which acts like a volumetric heat source and increases the internal energy of the fluid and thus stronger flow circulation creates. It is pragmatic from the figure that for $J=0$, there exists a clockwise flow pattern inside the cavity. The pattern of streamlines has no significant change due to rising Joule heating effect but the core of the vortex becomes slightly smaller. This phenomenon is seen for all $\lambda$. For $J=1$ and 2 , the pattern of the streamlines are approximately identical. It is also observed that due to creation of internal temperature, the fluid particle moves with greater velocity and the fluid flow covers the entire cavity in a circular pattern for all values of Joule heating parameter.

The isothermal lines for different values of Joule heating parameter and $\lambda$ have been expressed in the Figure 7(b). The Joule heating increases fluid temperature and reduces temperature differences. It is seen that as the value of $J$ rises the isothermal lines try to get crowded around the wavy surface of the enclosure and a thin boundary layer is developed near the left moving wall of the enclosure. But these lines distort in parabolic shape from the heated segment and start to gather at the bottom cold part of the cavity for all values of Joule heating number.

\subsection{Effect of average Nusselt number}

The variation of average Nusselt number $(\mathrm{Nu})$ of $\mathrm{Cu}-\mathrm{TiO}_{2}$-water hybrid nanofluid has been displayed in the Figure 8(a) along the hot wall against the wave number for different values of Richardson number at $H a=10, J$ $=1, \operatorname{Pr}=6.2$ and $\Phi=5 \%$. From the Figure 8(a), it is evident that with the increase of the value of $R i$ heat transfer rate increases. The rising of heat transfer is more effective on mounting the value of wave number. Because with the augmentation of $R i$ buoyancy effect enhances, so large amount of heat is transferred from the heated wall to the enclosure. In addition, due to corrugated surface $N u$ also increase. It is also found that increasing rate of heat transfer is much higher due to the mixing of nanoparticles in the base fluid which is known as hybrid nanofluid. Because the addition of solid nanoparticles to various fluids can increase the thermal conductivity and volume concentration of nanoparticles which interns improving the total heat transfer performance.

The effect of Hartmann number on heat transfer mechanism of hybrid nanofluid has been shown in the Figure 8(b) as a function of wave number at $R i=1.0, J=1.0, \operatorname{Pr}=6.2$ and $\Phi=5 \%$. It is found that the average Nusselt number decreases with increasing the Hartmann number. The average Nusselt number always becomes higher in absence of magnetic field $(\mathrm{Ha}=0)$. As magnetic field acts in opposition to the velocity it is evident from this figure that the strength of the circulation reduces with higher $H a$ and tends to retard the fluid motion in the bulk of the cavity. Due to rising and falling pattern of the wavy surface it is also observed that the average Nusselt number increases with increase of wave numbers.

In order to evaluate how the presence of Joule heating in the cavity affects the heat transfer rate of hybrid nanofluid along the hot wall the average Nusselt number $(\mathrm{Nu})$ of the fluid has been plotted as a function of number of wave for the value of $R i=1.0, \operatorname{Pr}=6.2, H a=10.0$ and $\Phi=5 \%$ in the Figure 8(c). It is observed that $N u$ reduces gradually while $J$ enhances slightly over the whole region for all values of $\lambda$ but it increases with the escalating values of undulation number $(\lambda)$. It is also noticed that $N u$ is always higher for the absence of Joule heating effect. It can be explained by the similar approach. 


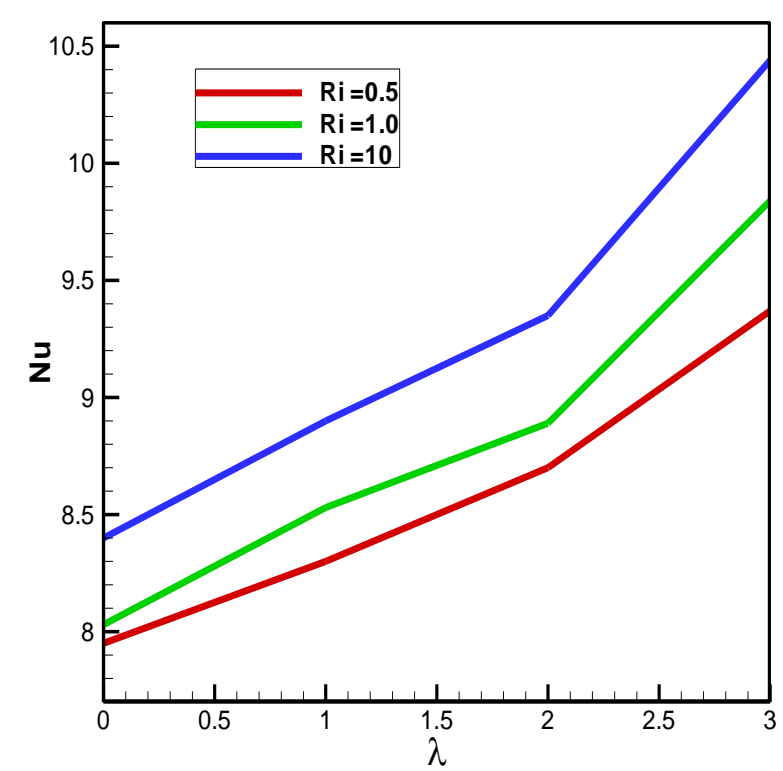

Figure 8(a): Average Nusselt number at various Richardson number

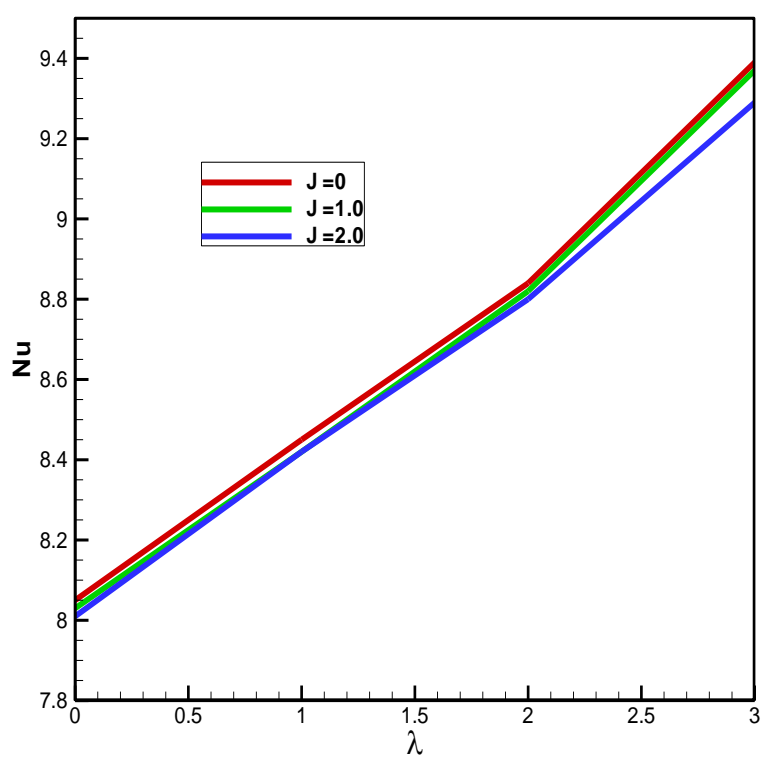

Figure 8(c): Average Nusselt number at various Joule heating

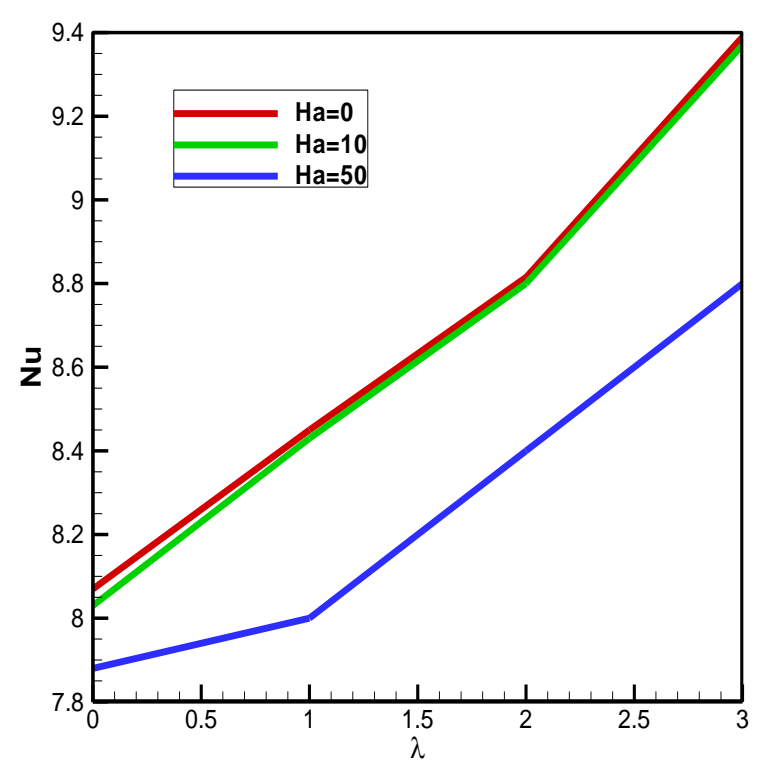

Figure 8(b): Average Nusselt number at various Hartmann number

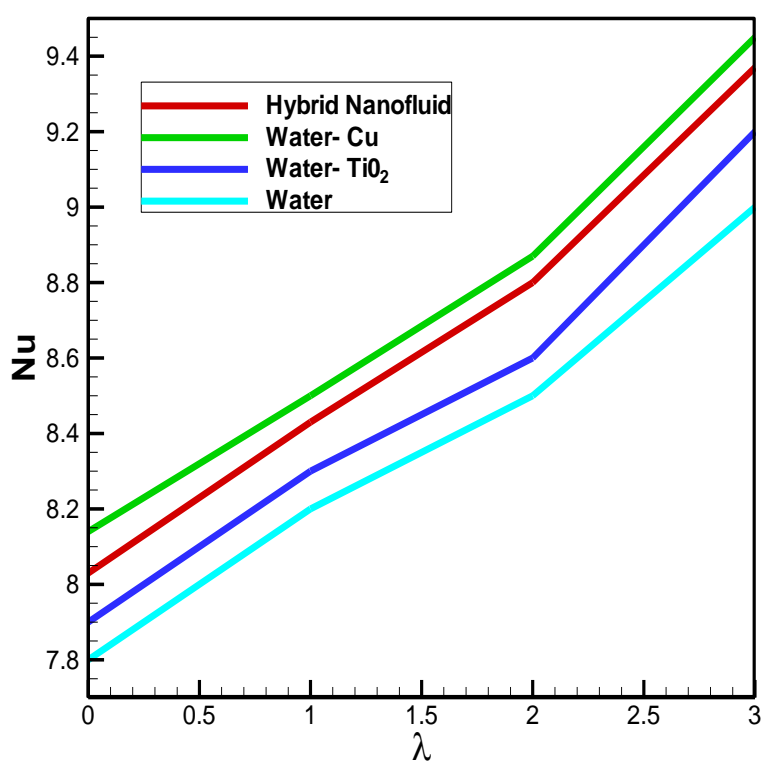

Figure 8(d): Average Nusselt number for different type of fluid

However, the distribution of mean Nusselt number $(\mathrm{Nu})$ for hybrid nanofluid, $\mathrm{Cu}$-water, $\mathrm{TiO}_{2^{-}}$water and base fluid water has been presented in the Figure 8(d) at $R i=1.0, \mathrm{Ha}=10.0, \mathrm{~J}=1.0, \mathrm{Pr}=6.2$ with $\Phi=5 \%$ solid volume fraction of nanoparticles. It has been reported that the enhancement of thermal conductivity dominates over viscous and inertia force in the fluid regime. Consequently it is also found that the increasing rate of heat transfer is much higher for hybrid nanofluid compared to the base fluid. The thermal conductivity of nanoparticles may be altered or changed by preparing hybrid nanofluid. Sometimes hybrid nanofluids become more effective heat transfer fluids than other single nanofluid or base fluid. The metallic nanoparticle like, $\mathrm{Cu}$ includes huge thermal conductivity but due to their stability and reactivity the use of metallic nanoparticles for nanofluid applications is limited. It can be predictable that the addition of metal nanoparticles (such as $\mathrm{Cu}$ ) into a nanofluid composed based on $\mathrm{TiO}_{2}$ nanoparticles, can enhance the thermo physical properties of nanofluid. 
But from this numerical research, it is found that the mixing properties of copper and $\mathrm{TiO}_{2}$ nanoparticles give a little bit smaller amount of convective heat transfer compare to $\mathrm{Cu}$-water nanofluid. Additionally, the rate of heat transfer for two different nanoparticles (hybrid) becomes higher than the $\mathrm{TiO}_{2}$-water nanofluid. In view of that, this result stimulates the researchers to examine other combination of different type nanoparticles, other models of thermos-physical properties of nanofluids and different volume fraction ratios of nanoparticles.

\section{Conclusion}

Hybrid $\mathrm{Cu}-\mathrm{TiO}_{2}$-water nanofluid flow in mixed convective lid-driven sinusoidal triangular enclosure in presence of MHD and Joule heating effect has been investigated numerically. Under these observations on varying several pertinent parameters, the following conclusions can be drawn:

- Undulated surface has a remarkable effect on the flow and temperature regimes. Rising values of wave number $(\lambda=3)$ enhances the heat transfer rate by $16.89 \%$ compared to flat surface $(\lambda=0)$. So, the corrugated lid driven cavity can be considered as an effective heat transfer system.

- For higher values the convective parameter $R i$ becomes dominating in the flow regime due to the influence of buoyancy force. For $\lambda=3$, approximately $11 \%$ heat transfer rate increases from the forced convection to free convection mode. It is also notable that the increment rate of heat transfer is optimum for the highest value of wave number.

- The cooling mechanism becomes significant in the situation when the magnetic field strength is very small. At $\lambda=3$, the decreasing rate of heat transfer is obtained about $12 \%$ for escalating values of Hartmann number from 0 to 50 , whereas for flat surface this rate becomes $2.23 \%$.

- The Joule heating parameter has tiny effect on streamlines and isothermal lines in the cavity. On elevating $J$ (from 0 to 2 ), the Nusselt number decreases by $1.06 \%$ for the highest undulation while a heat transfer reduction of $0.5 \%$ is observed for flat surface cavity.

- The enhanced rate of mean Nusselt number for hybrid nanofluid, $\mathrm{Cu}$-water and $\mathrm{TiO}_{2}$-water nanofluid are found as $4.11 \%, 5 \%$, and $2.2 \%$ respectively compared to base fluid. It is also perceived that the rate of heat transfer is much higher for hybrid nanofluid compared to some single nanofluid and base fluid.

It is concluded from the present numerical research that proper mixing of nanopartices may build the hybrid nanofluids very potential medium of heat transfer enhancement. Consequently, lot of research is still required in the field of stability, preparation, applications and characterization to overcome the challenges. It can also say that the prices of different nanoprticles are different. Analogously to the recent developments of the mono nanofluids, mixing properties of the advantages and disadvantages of nanoparticles are an announced job of hybridization. Furthermore, the suppliers of nanoparticles exhibit noticeable differences in costs of different nanoparticles categories. It can also say that the prices of different nanoprticles are different. For example the price of $\mathrm{Cu}$ nanoparticles is 10 times greater than $\mathrm{Al}$ nanoparticles. So it is better if one can achieve the required nanofluid properties on using expensive nanoparticle with minimum quantity, it will reduce the total cost of the research. This research is an effort to contribute the basics of thermal field of investigation. The obtained numerical results will inspire the researchers to examine other arrangement of dissimilar nanoparticles, extra models for the thermo-physical properties and other solid volume fraction ratios.

\section{References}

Agrouaz, Y., Bouhal, T., Jamil, A., Msaad, A.A., Kousksou, T., Mahdaoui, M., El Rhafiki, T. (2015): Effect of inclined wavy surface on heat transfer inside a rectangular cavity: Solar applications, 3rd International Renewable and Sustainable Energy Conference. https://dx.doi.org/10.1109/IRSEC.2015.7455006

Alam, Md. S., Khan, M.A.H., Saha, J., Nasrin, R. (2016): MHD mixed convection flow through a diverging channel with heated circular obstacle, AIP Conference Proceedings, 1754, 050020.

https://dx.doi.org/10.1063/1.4958411

Al-Amiri, A., Khanafer, K., Bull, J., Pop, I. (2007): Effect of sinusoidal wavy bottom surface on mixed convection heat transfer in a lid-driven cavity, International Journal of Heat and Mass Transfer, Vol. 50 No. 910, pp. 1771-1780. https://dx.doi.org/10.1016/j.ijheatmasstransfer.2006.10.008

Ali, M., Alim, M.A., Nasrin, R. and Alam, M.S. (2016): Effect of chemical reaction and variable viscosity on free convection MHD radiating flow over an inclined plate bounded by porous medium, AIP Conference Proceedings, 1754, 040009. https://dx.doi.org/10.1063/1.4958369 
Ali, M., Alim, M.A., Nasrin, R., Alam, M.S., Chowdhury, M.Z.U. (2017): Magnetohydrodynamic boundary layer nanofluid flow and heat transfer over a stretching surface, AIP Conference Proceedings, 1851, 020022. https://dx.doi.org/10.1063/1.4984651

Ali, M., Alim, M.A., Nasrin, R., Alam, M.S., Munshi, M.J.H. (2017): Similarity solution of unsteady MHD boundary layer flow and heat transfer past a moving wedge in a nanofluid using the Buongiorno model, Procedia Engineering, Vol. 194 (C), 407-413. https://dx.doi.org/10.1016/j.proeng.2017.08.164

Ali, M.M., Alim, M.A. and Nasrin, R. (2018): Effect of magnetic field on double diffusive natural convection flow in a closed chamber using nanofluid, AIP Conference Proceedings, 1980, 050020. https://dx.doi.org/10.1063/1.5044356

Arani, A.A.A., Sebdani, S.M., Mahmoodi, M., Ardeshiri, A., Aliakbari, M. (2012): Numerical study of mixed convection flow in a lid-driven cavity with sinusoidal heating on sidewalls using nanofluid, Superlattices and Microstructures, Vol. 51, No. 6, pp. 893-911. https://dx.doi.org/10.1016/j.spmi.2012.02.015

Babu, J.A.R., Kumar, K.K., Rao, S.S. (2017): State-of-art review on hybrid nanofluids, Renewable and Sustainable Energy Reviews, Vol. 77, pp. 551-565.

Ben-Cheikh, N., Chamkha, Ali J., Ben-Beya, B., Lili, T. (2013): Natural convection of water-based nanofluids in a square enclosure with non-uniform heating of the bottom wall, Journal of Modern Physics, Vol. 4, pp. 147159. https://dx.doi.org/10.4236/jmp.2013.42021

Botha, S.S., Ndungu, P., Bladergroen, B.J. (2011): Physicochemical properties of oil based nanofluids containing hybrid structures of silver nanoparticle supported on silica, Industrial \& Engineering Chemistry Research, Vol. 50, pp.3071-3077. https://dx.doi.org/10.1021/ie101088x

Brinkman, H.C. (1952): The viscosity of concentrated suspensions and solution, Journal of Chemical Physics, Vol. 20, pp. 571-581. https://dx.doi.org/10.1063/1.1700493

Bumataria, R.K., Chavda, N.K., Panchal, H. (2019): Current research aspects in mono and hybrid nanofluid based heat pipe technologies, Heliyon, 5, e01627. https://dx.doi.org/10.1016/j.heliyon.2019.e01627

Chamkha, A.J., Miroshnichenko, I.V., Sheremet, M.A. (2017): Numerical analysis of unsteady conjugate natural convection of hybrid water-based nanofluid in a semicircular cavity, Journal of Thermal Science Engineering Applications, Vol. 9, No. 4, 041004, 1-9. https://dx.doi.org/10.1115/1.4036203

Dechaumphai, P. (1999): Finite Element Method in Engineering, 2nd edition, Chulalongkorn University Press, Bangkok.

Gangawane, K.M., Manikandan, B. (2017): Mixed convection characteristics in lid-driven cavity containing heated triangular block, Chinese Journal of Chemical Engineering, 25 (10), 1381-1394.

https://dx.doi.org/10.1016/j.cjche.2017.03.009

Han, Z.H., Yang, B., Kim, S.H., Zachariah, M.R. (2007): Application of hybrid sphere/carbon nanotube particles in nanofluids, Nanotechnology, Vol. 18, No. 10, 105701. https://dx.doi.org/10.1088/0957$\underline{4484 / 18 / 10 / 105701}$

Ho, C.J., Huang, J.B., Tsai, P.S., Yang, Y.M. (2010): Preparation and properties of hybrid water based suspension of A12O3 nanoparticles and MEPCM particles as functional forced convection fluid, International Communications Heat Mass Transfer, Vol. 37, pp. 490-494.

https://dx.doi.org/10.1016/j.icheatmasstransfer.2009.12.007

Hossain, M.A., Gorla, R.S.R. (2013): Joule heating effect on magnetohydrodynamic mixed convection boundary layer flow with variable electrical conductivity, International Journal of Numerical Methods for Heat \& Fluid Flow, Vol. 23, No. 2, pp. 275-288. https://dx.doi.org/10.1108/09615531311293461

Ismael, M.A., Pop, I., Chamkha, Ali J. (2014): Mixed convection in a lid-driven square cavity with partial slip, International Journal of Thermal Sciences, Vol. 82, pp. 47-61. https://dx.doi.org/10.1016/j.ijthermalsci.2014.03.007

Jafari, M., Farhadi, M., Sedighi, K. and Fattahi, E.(2012): Effect of wavy wall on convection heat transfer of water-A12O3 nanofluid in a lid-driven cavity using lattice boltzmann method, IJE Transactions A: Basics, vol. 25, no. 2, pp. 165-176. https://dx.doi.org/10.5829/idosi.ije.2012.25.02a.06

Jan, S., Khojin, A.S., Zhong, W.H. (2007): Enhancement of fluid thermal conductivity by the addition of single and hybrid nano-additives, Thermochim Acta, Vol. 462, pp. 45-55. https://dx.doi.org/10.1016/j.tca.2007.06.009 Kumar, D.D., Arasu, A.V. (2018): A comprehensive review of preparation, characterization, properties and stability of hybrid nanofluids, Renewable and Sustainable Energy Reviews, Vol. 81, No. 2, pp. 1669-1689.

https://dx.doi.org/10.1016/j.rser.2017.05.257

Maxwell, J.C. (1873): A treatise on electricity and magnetism, Oxford, UK: Clarendon Press.

Muhammad, T., Hayat, T., Shehzad, S.A., Alsaedi, A. (2018): Viscous dissipation and Joule heating effects in MHD 3D flow with heat and mass fluxes, Results in Physics, Vol. 8, pp. 365-371. 
https://dx.doi.org/10.1016/j.rinp.2017.12.047

Nasrin, R. (2011): Aspect ratio effect of vertical lid driven chamber having a centered conducting solid on mixed magnetoconvection, Journal of Scientific Research, Vol. 3, No. 3, pp. 501-513.

https://dx.doi.org/10.3329/jsr.v3i3.7433

Nasrin, R. and Alim, M.A. (2009): Combined effects of viscous dissipation and temperature dependent thermal conductivity on MHD free convection flow with conduction and Joule heating along a vertical flat plate, Journal of Naval Architecture and Marine Engineering, Vol. 6, No. 1, pp. 30-40. https://dx.doi.org/10.3329/jname.v6i1.2648

Nasrin, R. and Alim, M.A. (2013): Free convective flow of nanofluid having two nanoparticles inside a complicated cavity, International Journal of Heat and Mass Transfer, Vol. 63, pp. 191-198.

https://dx.doi.org/10.1016/j.ijheatmasstransfer.2013.03.068

Nasrin, R., Alim, M.A. and Chamkha, Ali J. (2013): Effect of heating wall position on forced convection along two sided open enclosure with porous medium utilizing nanofluid, International Journal of Energy \& Technology, Vol. 5, No. 9, pp. 1-13.

Rashad, A.M., Chamkha, A.J., Ismael, M.A., Salah, T. (2018): Magnetohydrodynamic natural convection in a triangular cavity filled with a $\mathrm{Cu}-\mathrm{Al} 2 \mathrm{O} 3 /$ water hybrid nanofluid with localized heating from below and internal heat generation, Journal of Heat Transfer, Vol. 140, No. 7, 072502, pp. 1-13.

https://dx.doi.org/10.1115/1.4039213

Renewable and Sustainable Energy Reviews, Vol. 77, pp. 551-565.

Sarkar, J., Ghosh, P., Adil, A. (2015): A review on hybrid nanofluids: Recent research, development and applications, Renewable and Sustainable Energy Reviews, Vol. 43, pp. 164-177. https://dx.doi.org/10.1016/j.rser.2014.11.023

Sidik, N.A.C., Adamu, I.M., Jamil, M.M., Kefayati, G.H.R., Mamat, R., Najafi, G. (2016): Recent progress on hybrid nanofluids in heat transfer applications: A comprehensive review, International Communications in Heat and Mass Transfer, Vol. 78, pp. 68-79. https://dx.doi.org/10.1016/j.icheatmasstransfer.2016.08.019

Taylor, C., Hood, P. (1973): A numerical solution of the Navier-Stokes equations using finite element technique, Computer and Fluids, Vol. 1, pp. 73-89. https://dx.doi.org/10.1016/0045-7930(73)90027-3

Zahan, I., and Alim, M.A. (2018): MHD effect on solid fluid thermal conductivity ratio and wall thickness in a nanofluid filled enclosure, Journal of Engineering Mathematics \& Statistics, Vol. 2, No. 1, pp. 1-23.

Zahan, I., Nasrin, R. and Alim, M.A. (2018): MHD effect on conjugate heat transfer in a nanofluid filled rectangular enclosure, International Journal of Petrochemical Science and Engineering, Vol. 3, No. 3, pp. 114123. https://dx.doi.org/10.15406/ipcse.2018.03.00085

Zahan, I., Nasrin, R. and Alim, M.A. (2019): Hybrid nanofluid flow in combined convective lid-driven sinusoidal triangular enclosure, AIP Conference Proceedings, Vol. 2121, 070001, pp. 1-8.

https://dx.doi.org/10.1063/1.5115908 